\title{
The Variability of Neural Responses to Naturalistic Videos Change with Age and Sex
}

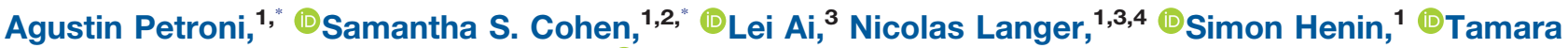 \\ Vanderwal, ${ }^{5}$ Michael P. Milham, ${ }^{3,6}$ and ${ }^{1}$ Lucas C. Parra ${ }^{1}$
}

DOI:http://dx.doi.org/10.1523/ENEURO.0244-17.2017

${ }^{1}$ Department of Biomedical Engineering, City College of New York, New York, NY 10031, ${ }^{2}$ Department of Psychology, the Graduate Center of the City University of New York, New York, NY 10016, ${ }^{3}$ Center for the Developing Brain, Child Mind Institute, New York, NY 10022, ${ }^{4}$ Methods of Plasticity Research, Department of Psychology, University of Zurich, 8050, Switzerland, ${ }^{5}$ Yale Child Study Center, New Haven, CT 06520, and ${ }^{6}$ Nathan Kline Institute for Psychiatric Research, Orangeburg, NY 10962

\begin{abstract}
Neural development is generally marked by an increase in the efficiency and diversity of neural processes. In a large sample $(n=114)$ of human children and adults with ages ranging from 5 to $44 \mathrm{yr}$, we investigated the neural responses to naturalistic video stimuli. Videos from both real-life classroom settings and Hollywood feature films were used to probe different aspects of attention and engagement. For all stimuli, older ages were marked by more variable neural responses. Variability was assessed by the intersubject correlation of evoked electroencephalographic responses. Young males also had less-variable responses than young females. These results were replicated in an independent cohort $(n=303)$. When interpreted in the context of neural maturation, we conclude that neural function becomes more variable with maturity, at least during the passive viewing of real-world stimuli.
\end{abstract}

Key words: Development; EEG; evoked responses; inter-subject correlation; naturalistic stimuli

\section{Significance Statement}

Naturalistic videos were used to probe response variability with EEG in a large developmental cohort. Our results are consistent with developmental theories positing that neural variability increases with maturation, and that neural maturation typically occurs earlier in females. These results differ from those observed with $\mathrm{fMRI}$, where an increase in stereotyped responses with age is observed during development.

\section{Introduction}

This study examines the relationship between the variability of neural responses and development. Over the course of development, the accuracy and stability of behaviors generally increase. This performance improvement is typically accompanied by a seemingly paradoxical increase in the variability of neural responses both within and across subjects (Grady, 2012; Dinstein et al., 2015). More-variable electroencephalographic (EEG) responses across trials, characterized by an increase in dimension-

Received July 7, 2017; accepted December 14, 2017; First published January $12,2018$.

Authors report no conflict of interest.

Author Contributions: MPM, LCP, SSC, and NL designed research; NL performed research; AP, SSC, NL, LA, and LCP analyzed data; AP, SSC, LA, $\mathrm{NL}, \mathrm{SH}, \mathrm{TV}, \mathrm{MPM}$, and LCP wrote the paper. ality and entropy, are associated with lower reaction time variability and higher recognition accuracy (Mcintosh et al., 2008). Neural variability often presents as an increase in the complexity of neural responses. This may be due in part to a developmental increase in the repertoire of possible brain states (Vakorin et al., 2013), and this increase in complexity may underlie the integration between distributed neural populations (Vakorin et al., 2011). EEG signal complexity becomes elevated in late adolescence and is also elevated in females relative to males at

This work was supported by the Defense Advanced Research Projects Agency (Contract W911NF-14-1-0157).

${ }^{*}$ A.P. and S.S.C. are co-first authors.

Acknowledgments: We thank Stefan Haufe for suggesting code to compute ISC using symmetrized between- and within-subject covariances.

Correspondence should be addressed to Lucas C. Parra, Department of 
this stage, indicating that females may attain mature brain functioning before males (Anokhin et al., 2000). Anatomic studies generally support the notion that females reach neural maturity before males (Giedd et al., 1999; Lenroot and Giedd, 2006; Lenroot et al., 2007; Marsh et al., 2008).

Neural variability does not always accompany proficient behavior, however. Both theta band coherence, a performance-monitoring measure, and behavior are more variable across trials in children (Papenberg et al., 2013). This suggests that neural variability does not always increase with maturation. For adults, the variability in "functional connectivity" between different networks measured with functional MRI (fMRI) is elevated during rest and decreases during a cognitive task. The reverse is true for children, whose brains become more variable during the task, and their performance is expectedly lower than adults (Hutchison and Morton, 2015).

Recently, responses to naturalistic narrative stimuli have been used to examine how variability in behavior and neural activity change with development. In these cases, variability is measured across subjects rather than within individuals because it is expected that if an individual has a less-variable neural response across repeated renditions of stimulus, their neural response will also be more similar to others who are responding to the same stimulus. Adults watch Sesame Street more similarly to each other than infants do, as assessed by where their eyes fixate (Kirkorian et al., 2012). Additionally, adults have more broadly similar neural responses to Sesame Street than children (Cantlon and Li, 2013). Although the neural responses of adults to Sesame Street correlate more with each other in many parietal and frontal regions, children correlate more strongly with each other in a specific region in the superior temporal cortex (Cantlon and $\mathrm{Li}$, 2013). Generally, from ages 18 to $88 \mathrm{yr}$, as humans age, responses to videos increase in variability (Campbell et al., 2015). Taken together, these studies demonstrate that neural variability changes with age. The nature of this relationship depends on multiple factors including the metric of neural variability, the developmental stage sampled, and the brain regions of interest.

Here, EEG was recorded from subjects with ages ranging from 5 to $44 \mathrm{yr}$ as they were presented with both naturalistic (Dmochowski et al., 2012, 2014) and conventional stimuli. To assess neural variability, the level of similarity across subjects was assessed with the intersubject correlation (ISC) of responses evoked by the stimuli. ISC of the EEG is indicative of attention, engagement, and memory in healthy adults (Dmochowski et al., 2014; Cohen and Parra, 2016; Ki et al., 2016; Cohen et al., 2017). We found that neural responses, indexed by ISC, become more variable with age. Among children, females have more-variable neural responses than males. This increase

\footnotetext{
Biomedical Engineering, City College of New York, 160 Convent Ave., New York, NY 10031. E-mail: parra@ccny.cuny.edu.

DOI:http://dx.doi.org/10.1523/ENEURO.0244-17.2017

Copyright (C) 2018 Petroni et al.

This is an open-access article distributed under the terms of the Creative Commons Attribution 4.0 International license, which permits unrestricted use, distribution and reproduction in any medium provided that the original work is properly attributed.
}

in variability is not due to a decrease in evoked response magnitude and was reproduced in two independent cohorts consisting of 114 and 303 individuals. These results are consistent with theories positing that development coincides with an increased repertoire of neural representations (Mcintosh et al., 2008), and the sex differences are consistent with the idea that young males are less neurally mature than young females (Giedd et al., 1999; Lenroot and Giedd, 2006; Lenroot et al., 2007; Marsh et al., 2008). Importantly, this is the first EEG study to report a measure of across-subject neural similarity with clear age and sex effects.

\section{Materials and Methods}

\section{Subjects}

In the main study, ages ranged from 6 to $44 \mathrm{yr}(n=114$, $14.2 \pm 8.0 \mathrm{yr}, 46$ females; see Fig. $1 A$ for a full age and sex distribution) as part of the Child Mind Institute-Multimodal Resource for Studying Information Processing in the Developing Brain (http://fcon_1000.projects.nitrc.org/indi/cmi_eeg/; Langer et al., 2017). In the replication study, ages ranged from 5 to $21 \mathrm{yr}(n=303,11.3 \pm 3.9 \mathrm{yr}, 135$ females; see Fig. $1 B$ for a full age and sex distribution). These data were obtained from the Child Mind Institute Healthy Brain Network (http://fcon_1000.projects.nitrc.org/indi/cmi_ healthy_brain_network/; Alexander et al., 2017). Both the main and replication study data come from publically available datasets. All experiments were performed in accordance with relevant guidelines and regulations. The study was reviewed and approved by the Chesapeake Institutional Review Board. All subjects presented with normal or corrected-to-normal vision.

\section{Stimuli}

Engaging, naturalistic videos were the primary stimuli. Specific videos were selected because they contained content relevant to social cognition, classroom anxiety, and attention. Three videos either featured educational content or depicted classroom scenarios: Fun with Fractals (Fract, MIT), a cartoon that explains fractals with examples (4 min 34 s), How to Improve at Simple Arithmetic (Arith, E-How), in which a math teacher in a typical educational setting explains addition and multiplication (1 $\mathrm{min} 30 \mathrm{~s}$ ), and Pre-Algebra Class (StudT, Pearson Education), showing an interaction between two students and a teacher (StudT, for student-teacher interaction) during math problem solving (1 min $40 \mathrm{~s})$. Two videos were clips from conventional cinema: Diary of a Wimpy Kid (Wimpy, Universal Pictures), a movie about a preteen starting middle school (1 min $57 \mathrm{~s}$ ), and Despicable Me (DesMe, Universal Pictures), which contains infant and toddler characters and emphasizes social interactions (2 min $51 \mathrm{~s}$ ). Although the main cohort contains data from all stimuli, the replication cohort only had three stimuli: Wimpy, Fract, and DesMe. The variability of the neural responses to these videos was measured across subjects using the ISC of evoked responses (see below). As a control condition, a "Rest" condition, during which subjects sat with their eyes closed for 4 min $20 \mathrm{~s}$, was also analyzed. This period establishes the baseline level of ISC, as no time-aligned 


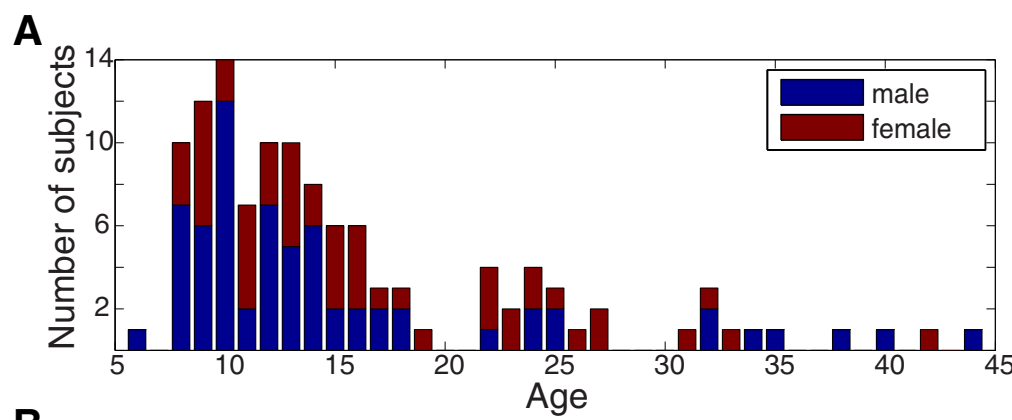

B

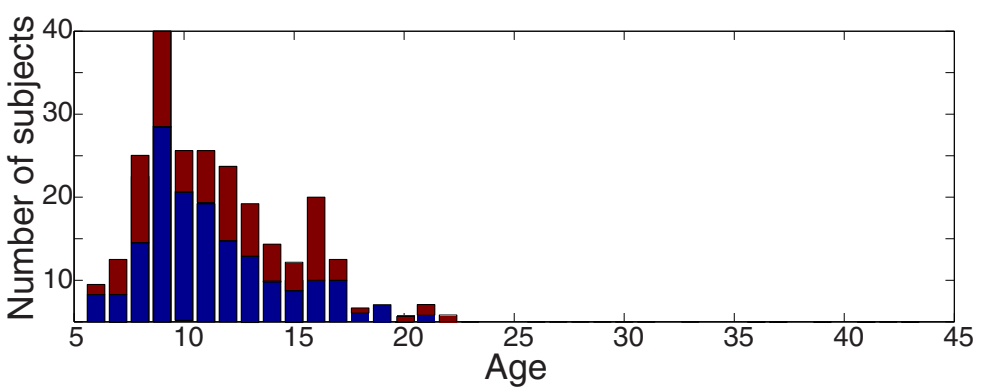

Figure 1. Age and sex distributions for the main study $(\boldsymbol{A})$ and replication study $(\boldsymbol{B})$. $\boldsymbol{A}$, Subjects in the main study $(n=114)$ had data for all of the stimuli. $\boldsymbol{B}$, Subjects in the replication study $(n=303)$ had only three stimuli (Wimpy, Fract, DesMe) and contribute to the results in Fig. 9.

stimulus entraining neural activity across subjects was presented. Finally, Flash, a stimulus condition without any narrative content, was used. During this stimulus, a blackand-white grating pattern that flashed at $25 \mathrm{~Hz}$ was presented for three minutes, thus synchronously stimulating neural activity across subjects (see Steady-state visual evoked potentials Methods section). This stimulus elicits steady-state evoked potentials (Vanegas et al., 2015) and was included to explore the extent to which ISC is driven by low-level evoked responses.

\section{Procedure}

While seated in a dimly lit room wearing an EEG net, subjects watched a series of short videos in a pseudorandom order. Stimuli were presented on a 17-inch CRT monitor (SONY Trinitron Multiscan G220, display dimensions $330 \times 240 \mathrm{~mm}$, resolution $800 \times 600$ pixels, vertical refresh rate of $100 \mathrm{~Hz}$ ). Note that some subjects did not experience all stimuli due to time limitations (Langer et al., 2017). Additionally, as explained below, poor data quality for some recordings caused additional data loss. For the replication study, only three conditions were used: Wimpy, Fract, and DesMe.

\section{EEG recordings and preprocessing}

EEG recordings were performed with an EGI Clinical Geodesic 128-channel system (Electrical Geodesic). Of the 128 channels recorded, 105 constituted the EEG recording and 11 represented electro-oculography (EOG) channels used for eye movement artifact removal. The remaining channels, mainly recording from the neck and face, were discarded. First, noisy channels were selected by visual inspection and replaced with by zero-valued samples, thus eliminating those channels' contribution in subsequent calculations of covariance matrices. Record- ings, initially at $500 \mathrm{~Hz}$, were then downsampled to 125 $\mathrm{Hz}$, high-pass-filtered at $1 \mathrm{~Hz}$, and notch-filtered between 59 and $61 \mathrm{~Hz}$ with a fourth-order Butterworth filter. Eye artifacts were removed by linearly regressing the EOG channels from the scalp EEG channels (Parra et al., 2005). Next, a robust principal components analysis (PCA) algorithm, the inexact augmented Lagrange multipliers methodp (Lin et al., 2013), was used to remove sparse outliers from the data following $\mathrm{Ki}$ et al. (2016). Briefly, robust PCA recovers a low-rank matrix, $\mathbf{A}$, from a corrupted data matrix $\mathbf{D}=\mathbf{A}+\mathbf{E}$, where some entries of the additive errors $\mathbf{E}$ may be arbitrarily large. Finally, individual recordings for some stimuli were discarded on the basis of visual inspection because they remained noisy after both automatic and manual noise removal. This was necessary because these subjects exhibited profound movement artifacts or the saline used for the recordings dried out. Despite these steps taken, the data overall appear to be of poorer quality than that collected in an electrically shielded room with conductive gel (saline was used here). The noise in the data may have led to the relatively low ISC values reported compared with previous studies (Ki et al., 2016; Cohen et al., 2017). However, it is unlikely that the noise contributed to our results, as under baseline conditions (Rest), there was no difference in power between the cohorts (see Results). All signal processing was performed offline using Matlab software (MathWorks).

\section{Intersubject correlation}

As variability is the inverse of similarity, we measured the similarity of evoked EEG responses across subjects. This approach has been used extensively to study concerted, intersubject changes in blood-oxygen leveldependent (BOLD) signal in fMRI (Hasson et al., 2004, 2009; Kauppi et al., 2010) and has been adapted to 
leverage the improved time resolution facilitated by EEG. To determine the neural similarity across subjects responding to the same stimulus (or in the same condition, in the case of Rest) the ISC of the EEG signal was computed as described previously (Dmochowski et al., 2012, 2014; Cohen and Parra, 2016; Ki et al., 2016). ISC assesses the level of correlation in the EEG across time among a group of subjects as they respond to the same stimulus. Larger ISC values imply more similarity in fast EEG responses across subjects ( $<1 \mathrm{~s})$. This indicates that the signals are more reliable due to decreased intersubject variability. It has also been found that subjects who pay more attention to the stimulus have higher ISC values (Ki et al., 2016). An advantage of the technique is that the stimulus need only be presented once to each subject, because evoked responses are compared across individuals. As repeated trials are unnecessary, responses are more similar to natural situations in which people experience uniquely presented novel stimuli. Additionally, in contrast to event-related potentials (ERPs), the technique can be applied to continuous and dynamic natural stimuli without the need for specific event markers (Ben-Yakov et al., 2012). As such, the approach is data driven both spatially and temporally - spatially, because the data from the subjects determines the best combination of electrodes (which are spatially distributed across the scalp and therefore may correspond with different anatomic regions) that maximize the correlation across subjects, and temporally, because the ultimate correlation values are determined by the temporal fluctuations in the EEG signals. In contrast, a more traditional approach to EEG data analysis would be to select electrodes that have previously been shown to elicit a certain effect (or ERP) and measure event-locked responses from these electrodes. We are not taking this approach. Rather, the electrodes that we chose and the time periods that maximize correlation are determined directly by the data itself.

ISC utilizes correlated component analysis to identify linear combinations of EEG electrodes that capture most of the correlation across subjects (Dmochowski et al., 2012). Correlated component analysis is similar to PCA except that rather than maximizing variance within one dataset, it selects projections, $\boldsymbol{v} \in \boldsymbol{R}^{D}$, where $D$ is the number of electrodes, that maximize the correlation between multiple datasets. These projections can be thought of as virtual sensors (or component sources) of activity that are optimized to capture most of the correlation between subjects. They are the eigenvectors of $R_{W}^{-1} R_{B}$, where $R_{W}$ is the average within-subject covariance, $1 / N \sum_{k} R_{k k}$, and $R_{B}$ is the average between subjects cross-covariance, $1 / N(N-$ 1) $\sum_{k|,| \neq k} R_{k l}$, and $R_{k l}=\sum_{t}\left[x_{k}(t)-\bar{x}_{k}\right]\left[x_{l}(t)-\bar{x}_{l}\right]^{T}$ measures the cross-covariance of all electrodes in subject $k$ with all electrodes in subject $I$. Vector $x_{k}(t)$ is the scalp voltages at time $t$ in subject $k$, and $\bar{x}_{k}$ is their mean value in time.

Following previous research, we use the three components, or eigenvalues of $R_{W}^{-1} R_{B}$, that represent the largest fraction of the correlation across subjects. These components can be optimized for all subjects together, or for a subset of the entire cohort. The subsets used in this paper are stimulus (Wimpy, DesMe, Fract, Arith, StudT, Flash, and Rest), age group (young vs. old), sex (male vs. female), and sex and age group combined (young-male, youngfemale, old-male, and old-female). ISC components are computed within subsets of the entire sample to examine potential differences in the spatial distribution of activity across groups, although the spatial patterns are largely consistent (Fig. 7).

To calculate the ISC for individual subjects as they respond to the same condition as their peers, the correlation between each individual's EEG responses and the responses from all other individuals is calculated (Cohen and Parra, 2016; Ki et al., 2016). The ISC values reported throughout the paper are this measure of how well each individual correlates with the others. The projections, $\boldsymbol{v} \in \boldsymbol{R}^{D}$, used to compute this subject-specific ISC value are computed either across all subjects or within the subgroups listed above (divided by either stimulus, age, sex, or age + sex). The ISC for each subject is therefore

$$
C_{i k}=\frac{v_{i}^{T} R_{b, k} v_{i}}{V_{i}^{T} R_{w, k} v_{i}}
$$

where $R_{b, k}=1 /(N-1) \sum_{l, l \neq k}\left(R_{k l}+R_{l k}\right)$ and $R_{w, k}=1 /$ $(N-1) \sum_{1, l \neq k}\left(R_{k k}+R_{l \mid}\right)$. ISC for subject $k$ is therefore $\sum_{i=1}^{3} C_{i k}$. A simplified template for the code to compute the correlated components and the ISC for individual subjects is available at http://www.parralab.org/isc/

\section{Steady-state visual evoked potentials}

To determine the strength of low-level sensory evoked responses across individuals, we leveraged the steady state visual evoked potential (SSVEP) paradigm (Flash) that was part of the data collection effort (Langer et al., 2017). Stimulus and analysis followed established techniques (Vanegas et al., 2015). Briefly, the stimulus consisted of four circular "foreground" stimuli (vertical grating, radius $2^{\circ}$ ) that were flickered on and off at $25 \mathrm{~Hz}$ and embedded in a static "background" grating, which is known to generate reliable SSVEPs (Vanegas et al., 2015). This stimulus was presented in trials of 2.4-s duration with intertrial intervals of $1 \mathrm{~s}$, which included a fixation cross presented for $0.5 \mathrm{~s}$. The stimuli were presented in several conditions that varied in their contrast and in the phase relationship between the foreground and the background. A total of 128 trials were present (12 conditions total: four foreground contrasts: $0 \%, 30 \%, 60 \%$, and $100 \%$, and three background conditions: parallel phase, orthogonal phase, and no surround stimuli). Artifacts were rejected by removing trials for which the power (or absolute value) of any electrode exceeded more than three standard deviations above the mean. EOG activity was regressed out of the EEG, as described above. The initial $200 \mathrm{~ms}$ of each trial was removed to eliminate the onset of the visual evoked response. Data were Fourier transformed for each trial, and power in a $0.5-\mathrm{Hz}$ bin surrounding the $25-\mathrm{Hz}$ band was extracted and then averaged across all trials, regardless of condition (thus ignoring details of the foreground-background interaction). Because the EEG activity 
measured with this paradigm is known to be dominated by primary visual cortex (V1) responses, power was averaged over the five most relevant occipital electrodes (01-O5; (Vanegas et al., 2015).

\section{Dimensionality of EEG responses}

To gain a sense of the dimensionality of the EEG responses across subjects, the eigenvalue spectrum was extracted from each subject's covariance matrix (covariance between all electrodes measured across time). These covariance matrices measure the correlation between electrodes for each subject. The sum of the eigenvalues represents the overall power in the data. To assess the dimensionality of the data, lines were fitted to the log-log plot of the eigenvalue spectrum of each subject's covariance matrix. A shallower slope of the linear fit indicates that the there is appreciable power over a larger number of dimensions. Two-way ANOVAs and subsequent post hoc $t$ tests were employed to compare power and the slopes of these linear fits for each age and sex group both across all stimuli and within each stimulus.

\section{Results}

We sought to determine whether and how the variability of EEG differs across age and sex in children and adults ranging from 6 to $44 \mathrm{yr}$ of age. To assess the variability in EEG signals across subjects, the ISC between individuals and their peers was assessed in response to both naturalistic videos and artificial stimuli. ISC can be thought of as a measure of the similarity of neural responses (Dmochowski et al., 2012). If subjects respond more similarly to their peers, they will have a larger ISC value, which indicates that they have a less-variable neural response.

\section{ISC varies between stimuli}

ISC is a stimulus-driven measure of attention ( $\mathrm{Ki}$ et al., 2016), because neural responses are more correlated across subjects when they naturally attend to a stimulus than when they are engaged in a dual task. It is therefore expected to be indicative of varying levels of engagement (Cohen et al., 2017). A one-way ANOVA determined that ISC significantly depended on the stimulus $(F(7)=78.26$, $p=10^{-68}$; mean \pm STD ISC values: Wimpy: $0.053 \pm$ 0.036; DesMe: $0.035 \pm 0.023$; Arith: $0.019 \pm 0.013$; Fract: $0.026 \pm 0.016$; StudT: $0.012 \pm 0.009$; Flash: $0.030 \pm$ 0.019 ; Rest: $0.001 \pm 0.004$ ), indicating that the stimuli significantly varied in engagement level (Cohen et al., 2017). It is worth noting that these ISC values are relatively low compared with previous research (Cohen and Parra, 2016; Ki et al., 2016). There are two factors that contribute to this discrepancy: the lower production quality and therefore engagement level elicited by these stimuli and the relatively poor quality of the EEG data (see Methods). Note also that ISC for EEG is generally lower than ISC of fMRI (e.g. Lahnakoski et al., 2017), which has a slower time scale and higher signal-to-noise ratio, factors that can both contribute to higher correlations (Haufe et al., 2017). As expected, ISC in the Rest condition was not significantly different from zero ( $t$ test, $t(45)=0.52, p=$ 0.4 ), confirming the notion that ISC reflects stimulusinduced correlations (Dmochowski et al., 2012). A one- way ANOVA was therefore performed on all stimuli excluding Rest, confirming that ISC strongly varies between stimuli $\left(F(6)=71.70, p=10^{-55}\right)$. Tukey post hoc pairwise comparisons revealed that ISC was significantly stronger when evoked by the qualitatively more engaging stimuli (Wimpy and DesMe), than it was for educational videos (Arith, Fract, StudT; Tukey post hoc pairwise comparisons between each pair of videos, Tukey's HSD: $p<10^{-4}$ ). Among the more engaging videos from conventional cinema, Wimpy, a movie trailer for the feature film "Diary of a Wimpy Kid," evoked a higher level of neural similarity than DesMe, a scene from the animated film "Despicable Me" (Tukey's HSD: $p=10^{-7}$ ). Among the relatively lessengaging educational videos, Fract elicited the highest level of ISC, which was significantly higher than StudT (Tukey's HSD: $p=10^{-6}$ ), but not Arith (Tukey's HSD: $p=$ $0.2)$. Interestingly, Arith elicited a level of ISC similar to Flash (Tukey's HSD: $p=0.5$ ), and the level of ISC elicited by Flash was significantly higher than StudT (Tukey's HSD: $p=10^{-7}$ ).

\section{ISC decreases with age}

We hypothesized that neural similarity changes with age and therefore examined the correlation between ISC and age. Here, ISC is computed in individuals by measuring the extent to which each subject correlated with the other people in the same stimulus condition. For all of the stimuli excluding Rest, there was a negative relationship between age and ISC (all $r=-0.68 \pm 0.09$, all $p<10^{-10}$, false discovery rate corrected following Benjamini and Hochberg (1995) (Fig. 2). ISC did not vary with age during Rest $(r=-0.10, p=0.5, n=46)$. This was expected, since Rest contained no stimulus to drive EEG signal similarly across subjects.

These results indicate that ISC decreases with age. However, most of the subjects in the main study were from the lower half of the age distribution (Fig. 1A). Because the components used to measure ISC are optimized to capture the correlation across all subjects, the components may have been biased by these younger subjects, who constituted a majority of the sample. The cohort was therefore divided into two age groups of equal size to eliminate this potential measurement bias. The median split resulted in groups whose ages ranged from 6 to 14 (mean age $10.74 \pm 2.03 \mathrm{yr}$ ) and 15 to 44 (mean age $23.65 \pm 8.04 \mathrm{yr}$ ). The ISC was then recomputed from components extracted separately in each group. A twoway ANOVA with factors of age and stimulus revealed that ISC was significantly modulated by both stimulus (all excluding Rest, $\left.F(5,393)=63.64, p=10^{-47}\right)$ and age $\left(F(1,393)=335.46, p=10^{-53} ;\right.$ Fig. $\left.3 A\right)$. For all stimuli, ISC was much higher in the younger age group.

\section{ISC is elevated in males}

Sex is an important factor that influences the developmental trajectory of the human brain (Giedd et al., 1999; Lenroot and Giedd, 2006; Lenroot et al., 2007; Marsh et al., 2008). We therefore explored the relationship between sex and ISC. A two-way ANOVA with factors of sex and stimulus (excluding Rest) revealed main effects for both $\operatorname{sex}\left(F(1,393)=53.11, p=10^{-12}\right)$ and stimulus $(F(5$, 

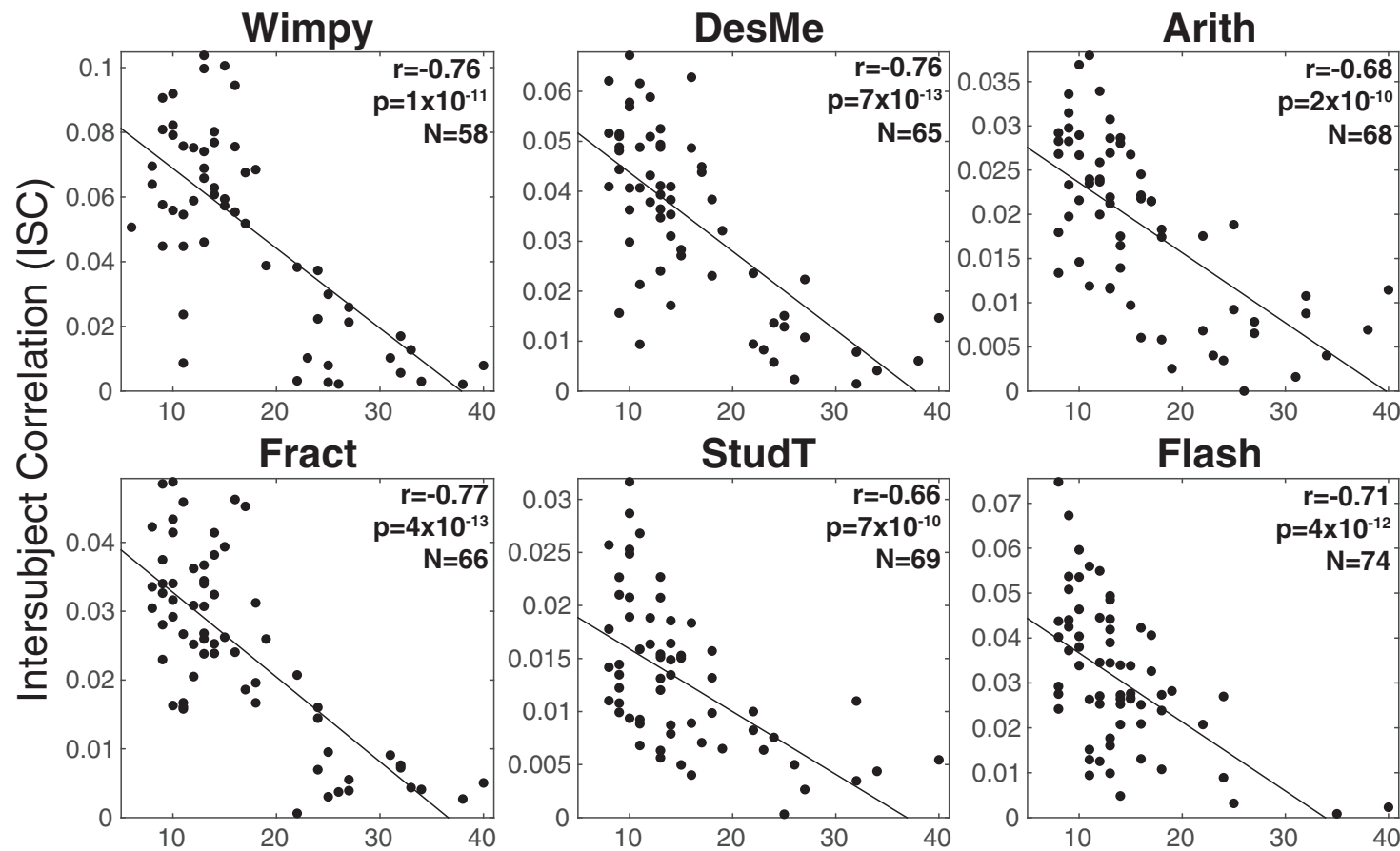

StudT

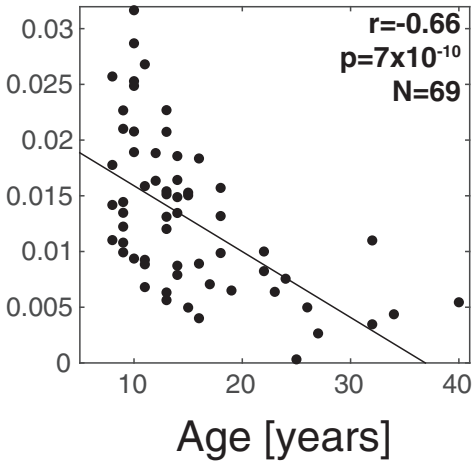

Flash

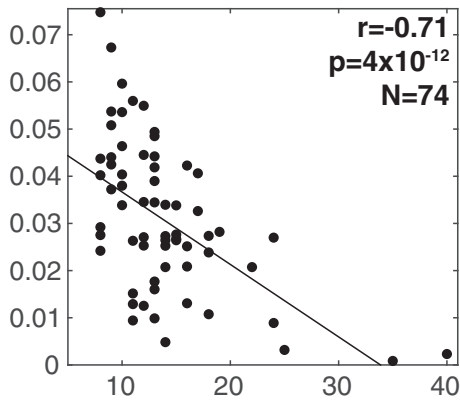

Figure 2. Neural similarity, measured as the ISC of neural activity, decreased with age. Correlation values ranged from $r=-0.58$ to $r=-0.78$, indicating a consistent relationship between maturity and neural variability. ISC was computed for each individual by correlating neural responses from individual subjects with the neural responses from all other subjects for that stimulus (regardless of age and sex).

393) $=30.12, p=10^{-26}$; Fig. 3B). Tukey's post hoc tests revealed that ISC was consistently higher in males for all stimuli except for Flash, where it was marginally significant (Flash: $p=0.06$; Wimpy: $p=0.03$; DesMe: $p=10^{-6}$; Arith: $p=0.003$; Fract: $p=10^{-4}$; StudT: $p=10^{-4}$ ). To examine whether the sex difference depended on age, the data were separated into four groups with the same age division between 14 and $15 \mathrm{yr}$ as above (young-male, young-female, old-male, old-female). ISC was measured within each group and averaged across all stimuli available for each subject to ensure sufficiently large sample sizes (excluding control conditions, Flash and Rest; Fig. 4). A two-way ANOVA with sex and age as factors confirmed the age effect $\left(F(1,87)=98.85, p=10^{-16}\right)$, and the
A

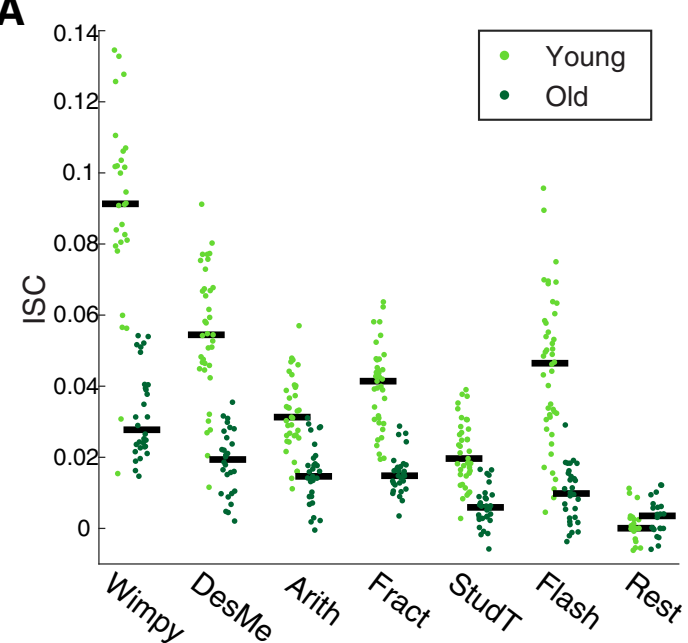

B

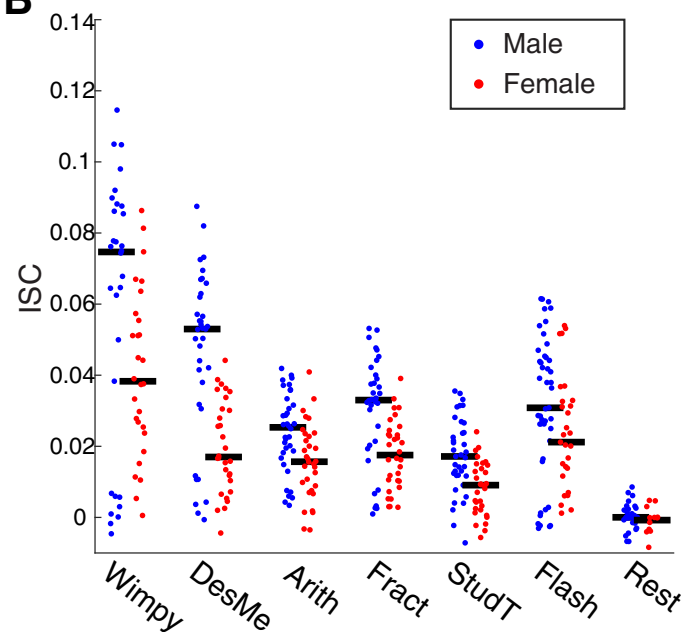

Figure 3. ISC, a measure of neural similarity, was consistently higher among younger ages and males. $\boldsymbol{A}$, Across all stimuli, ISC was higher for younger subjects (6-14 yr, light green) than older subjects (15-44 yr, dark green). $\boldsymbol{B}$, Across all stimuli, ISC was higher for males (blue) than females (red). For both $\boldsymbol{A}$ and $\boldsymbol{B}$, ISC was computed separately within each age and sex group. Black lines indicate the median. 


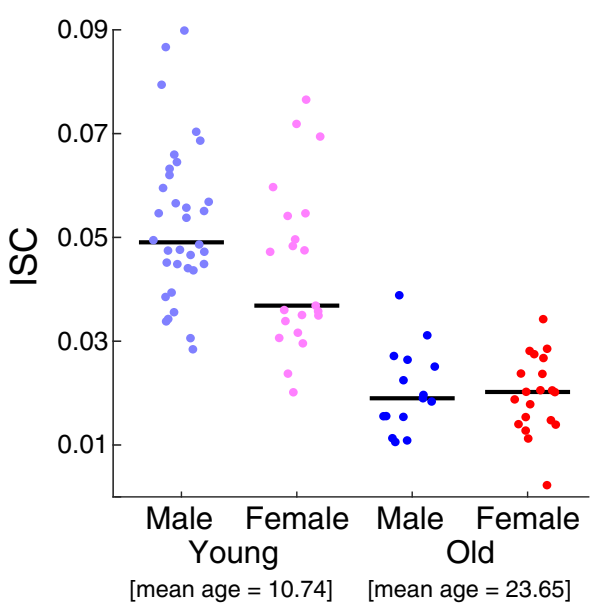

Figure 4. Sex differences in the young do not exist in the old. Young males were more neurally similar to each other than young females. This sex difference is absent in the older group. Here, ISC was computed within each sex and age group separately and averaged across all stimuli except for Flash and Rest. Black lines indicate the median.

sex effect was marginally significant $(F(1,87)=3.83, p=$ $0.05)$. A direct comparison between the sexes in each age group revealed that the sex effect was marginally significant among the young ages $(t(53)=2.02, p=0.05,6-14$ yr), but not present for the old ages $(t(33)=0.28, p=0.8$, $15-44 \mathrm{yr})$.

\section{The effect of age on ISC is not due to evoked response difference}

The relationships between ISC, age, and sex may be partially driven by the reduction of evoked response magnitude with age (Goodin et al., 1978; Tomé et al., 2015). Although correlation, which ISC measures, is theoretically independent of magnitude, it is possible that a decrease in magnitude corresponds with a decrease in the signalto-noise ratio, which would result in a smaller ISC. The magnitude of evoked responses was therefore assessed with the Flash stimulus, which elicited SSVEPs (see Methods). SSVEP magnitude weakly declines with age $(r=$ $-0.22, p=0.02, n=109$; Fig. $5 A$ ) and a two-way ANOVA with age and sex as factors (the same age/sex groups as Fig. 4) found the age effect to be marginally significant $(F(1106)=4.00, p=0.05$; Fig. $5 B)$. There was no significant relationship between sex and SSVEP strength $(F(1106)=3.3, p=0.08)$.

Because both SSVEP amplitude and ISC decrease with age, we reasoned that SSVEPs could be used to factor out the effect of evoked response strength (Goodin et al., 1978; Tomé et al., 2015). Indeed, ISC and SSVEP amplitude are correlated across subjects $(r=0.41, p=0.0001$, $n=84$; Fig. $6 A$ ). To control for the effect of evoked
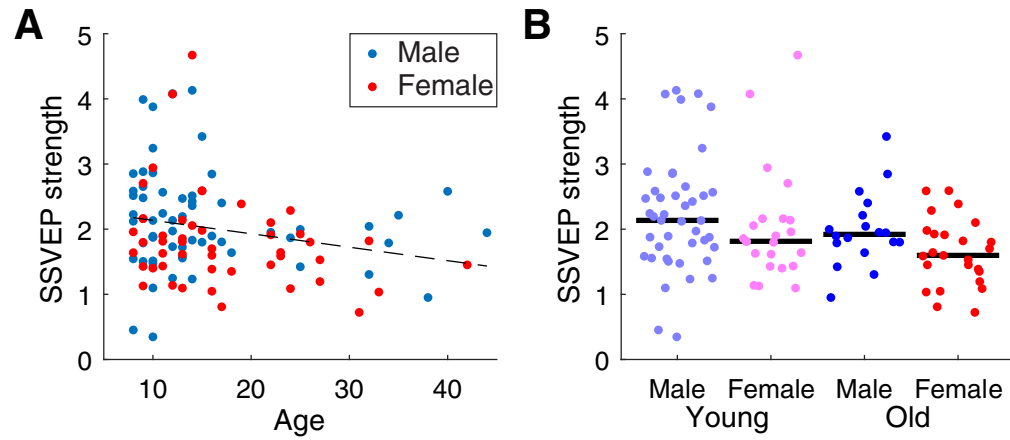

Figure 5. SSVEP magnitude depended on age, but not on sex. A, SSVEP strength was weakly correlated with age across subjects, but it was no different between males and females. $\boldsymbol{B}$, SSVEP strength was no different between males and females. Black lines indicate the median.
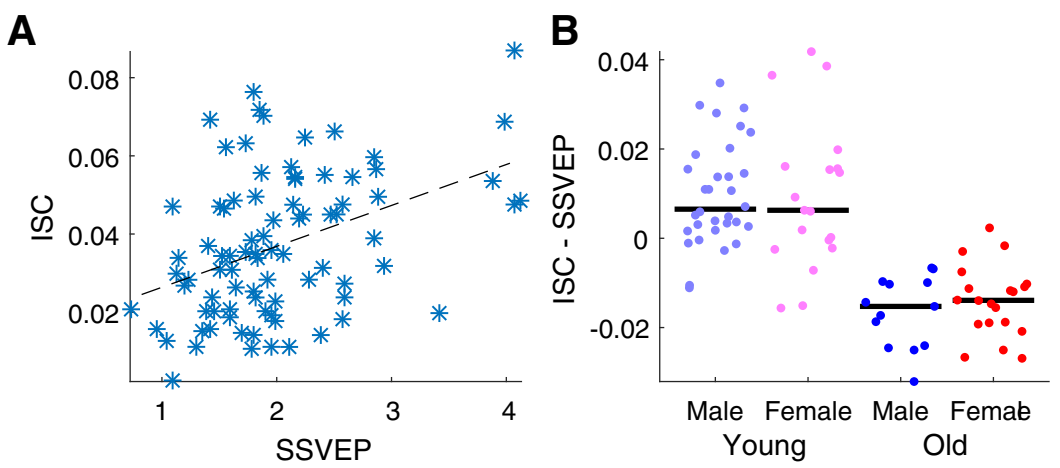

Figure 6. Relationship between ISC magnitude and SSVEP strength. $\boldsymbol{A}$, SSVEP strength, a measure of the magnitude of evoked responses, correlated with ISC strength, calculated using all stimuli except for Flash and Rest. $\boldsymbol{B}$, Comparison of ISC strength after SSVEP magnitude was regressed out (ISC - SSVEP) between males and females in the young and old age groups. Although there was a significant difference between the age groups, a difference between the sex groups was not present. Black line indicates the median. 

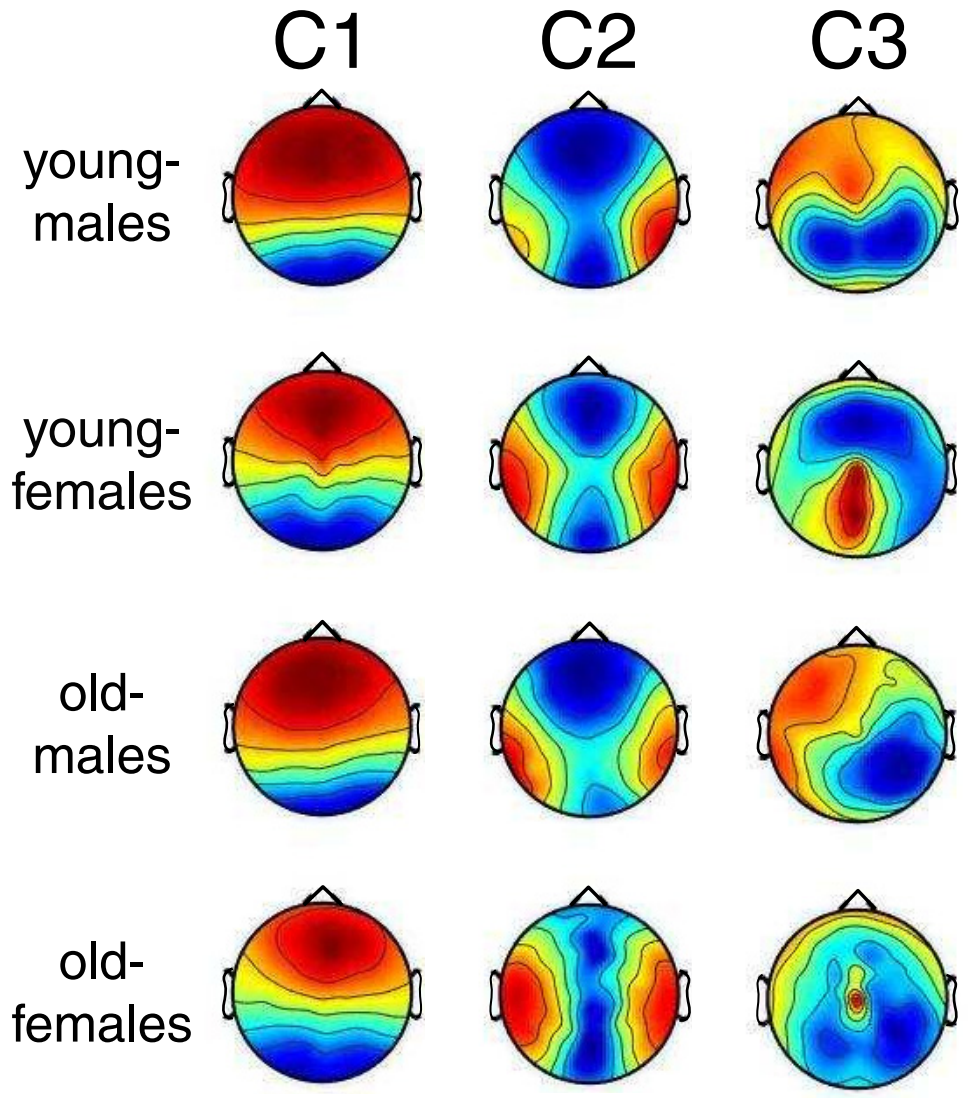

Figure 7. Spatial distributions corresponding to the three strongest components of ISC (C1-C3). Red and blue colors indicate positive and negative correlation of the voltages on the scalp surface with the component activity. These maps are unitless due to an arbitrary scale on the projection vectors. Here, the projections have been computed separately for the combination of the two sex and age groups. As the scalp topographies were relatively consistent across the groups, the differences in ISC across these groups were not due to differences in the spatial topography of correlation within the group.

response strength, each individual's SSVEP amplitude was linearly regressed against ISC, and the portion that could be explained by the SSVEP was subtracted (ISC calculated within the same age/sex group as Fig. 4). A two-way ANOVA with age and sex as factors revealed that this residual ISC still significantly varies with age $(F(1,81)=$ $\left.85.49, p=10^{-14}\right)$, but does not vary with $\operatorname{sex}(F(1,81)=0.08$, $p=0.8$; Fig. 6B). Additionally, the sex effect is no longer present in the younger group when SSVEP strength is controlled for $(t(49)=0.11, p=0.9)$. The lack of a sex effect may mean that the relationship between sex and neural variability is due in part to evoked response magnitude, but the lack of an effect may also result from the reduced number of subjects for which SSVEP magnitude was available: 84 versus 114. Regardless, neural variability, as assessed by ISC, does increase with age, regardless of the strength of evoked responses.

\section{Correlated component topographies are similar across age and sex groups}

ISC was measured using components of the EEG that maximize correlations between subjects. These components are linear combinations of electrodes and can be thought of as virtual sensors (see Methods). To determine whether the spatial distribution of the corresponding ac- tivity differed across groups, the "forward" model, which represents how the components look on the surface of the scalp, was computed for the largest three components that were used to compute ISC (Parra et al., 2005). These component topographies were very similar across all age/sex groups for the strongest two components, C1 and $\mathrm{C} 2$ (Fig. 7, minimum cosine similarity 0.97 for $\mathrm{C} 1$ and 0.78 for C2). The third component (C3) was less similar across the groups (cosine similarity $0.89-0.31$ ), but it also constituted a much weaker portion of the ISC $(\mathrm{C} 1=0.016 \pm 0.009, \mathrm{C} 2=0.008 \pm 0.005$, and $\mathrm{C} 3=$ $0.004 \pm 0.003$, computed as in Fig. 4 and averaged across all subjects and stimuli). Thus, for the most part, differences in ISC between age and sex groups were not due to differences in the spatial distribution of neural activity across these groups.

\section{Dimensionality of EEG responses}

To determine whether the differences in ISC across groups was due to diverse responses across subjects or to more highly dimensional responses within subjects, the eigenvalue spectra of the EEG covariance matrices were analyzed (Fig. 8). The sum of these spectra represents the overall power of the data. In general, the younger age group (using the same median split as above) had more power 

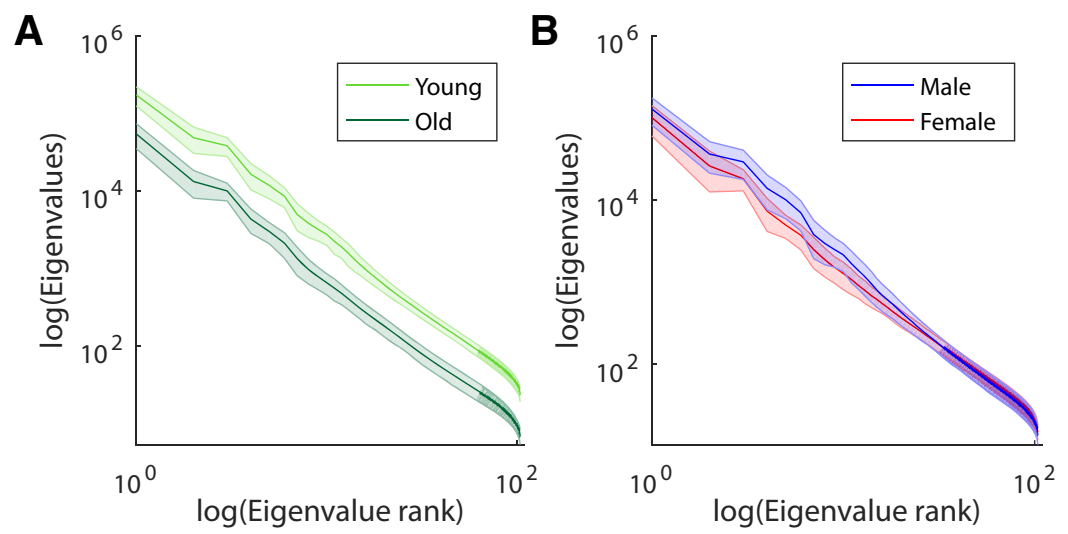

Figure 8. Eigenvalue spectra of the average covariances for each demographic group. Eigenvalues measure the power of the signal in principal components of the EEG (correlated across time for each stimulus). Each curve is the average eigenvalue spectrum for each group averaged across all stimuli and subjects. $\boldsymbol{A}$, Young subjects have more power than old subjects in all dimensions. This is represented by the upward shift in their average eigenvalue spectrum. $\boldsymbol{B}$. The eigenvalue spectrum of females has a shallower slope than that for males, indicating that they have a more diverse set of neural responses.

than the older age group across all stimuli $(F(1438)=$ $452.13, p=10^{-69}$ ). This suggests that there was more overall power in the EEG of the young group. This power difference was present only during the stimuli, not during rest $(t(44)=0.6, p=0.5)$, suggesting that younger subjects have stronger stimulus-driven evoked responses. To assess the dimensionality of the EEG responses, a linear model was fitted to each subject's eigenvalue spectrum (see Methods), and the slopes were compared between the groups. A difference in dimensionality is reflected by a difference in this slope, with a shallower slope indicating that there is a higher number of dimensions with appreciable signal. The slopes of the linear fit did not differ across the age groups (across all stimuli: $F(1438)=2.74$, $p=0.1$ ). This suggests that the stimulus-evoked responses are not inherently of higher dimensionality in the young. For the sex comparison, males had higher overall power (across all stimuli: $F(1438)=71.25, p=10^{-16}$ ), for all stimuli and not during rest $(t(44)=0.9, p=0.3)$. Here females had a shallower slope than males (across all stimuli: $\left.F(1438)=152.12, p=10^{-30}\right)$. This suggests a greater complexity of responses within females.

\section{Replication of results}

To confirm these findings, the results were replicated in an independent cohort $(n=303)$ with a reduced stimulus set: Wimpy, Fract, and DesMe. Replicating the results above, ISC also decreased with age in this cohort (Wimpy: $r=-0.44, p=10^{-14}, n=276$; Fract: $r=-0.37, p=$ $10^{-10}, n=270 ;$ DesMe: $r=-0.41, p=10^{-12}, n=281$; Fig. 9A). A two-way ANOVA with age and condition as factors revealed that ISC is modulated by age $(F(1799)=$ 35.33, $\left.p=10^{-9}\right)$ and stimulus $(F(2799)=272.903, p=$ $10^{-91}$; Fig. 9B). A two-way ANOVA with sex and stimulus as factors revealed that ISC was also significantly modulated by $\operatorname{sex}(F(1823)=11.12, p=0.0009$; Fig. $9 C)$ and stimulus $\left(F(2823)=430.95, p=10^{-129}\right)$. Finally, a twoway ANOVA that divided the data across age and sex groups and averaged ISC across stimuli replicated the main effect of age $\left(F(1291)=17.68, p=10^{-5}\right)$ and did not find an effect of $\operatorname{sex}(F(1291)=2.59, p=0.1$; Fig. $9 D)$.
Follow-up analyses that examined a potential sex difference in ISC in each age group revealed that the difference in ISC was present at young ages $(t(224)=2.29, p=0.02$, $5-14 \mathrm{yr})$, but not old ages $(t(67)=0.59, p=0.6,15-21 \mathrm{yr})$. When the median was calculated according to the median of the replication distribution (split at 10/11 years; see Fig. 1B for age distribution), the above results were unchanged. In summary, all results from the main experiment were replicated in this independent cohort.

\section{Discussion}

The present work demonstrated age- and sex-related variability among individuals with respect to their neural responses to complex naturalistic stimuli. Specifically, ISC was significantly correlated with age for both naturalistic videos and artificial visual flashes. Younger subjects $(6-14 \mathrm{yr})$ exhibited less-variable neural responses than older subjects (15-44 yr). A parallel finding revealed that young males exhibited more similar responses to the stimuli than young females, a difference that was present only in the younger cohort. These age and sex effects may result from neural development, consistent with the notion that neural maturation occurs later in males than in females (Lenroot et al., 2007; Marsh et al., 2008; Mous et al., 2017). A quantitative analysis of the spatial distribution of the correlated activity revealed that the observed age and sex differences are largely driven by the same neural components, lending more weight to the idea that the observed differences in age and sex stem from a common developmental feature. Finally, a replication study with 303 participants yielded similar results.

A possible confound for the present results is that the neural correlations found across subjects are due to correlations in overt behaviors such as eye movements. However, it is unlikely that eye movements follow the same developmental trajectory as neural responses, because eye movement trajectories evoked by videos actually become more similar with age (Kirkorian et al., 2012). Thus, although the gaze patterns evoked by videos seem to converge with maturity, potentially driving similar bottom-up neural processes, neural similarity as measured by ISC, decreases 
A
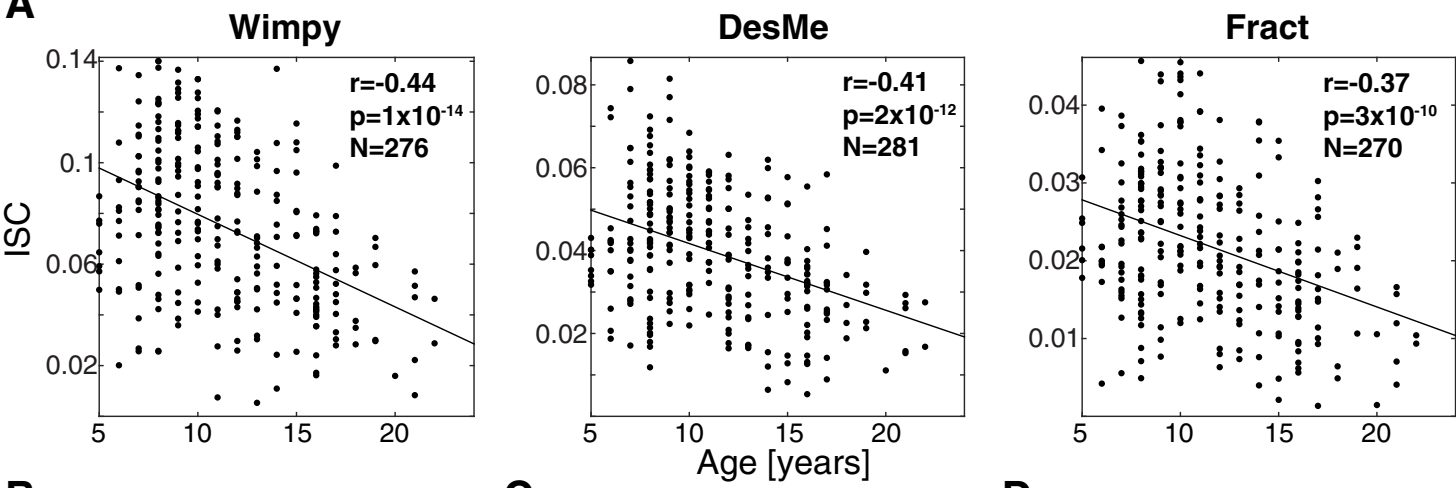

B

C
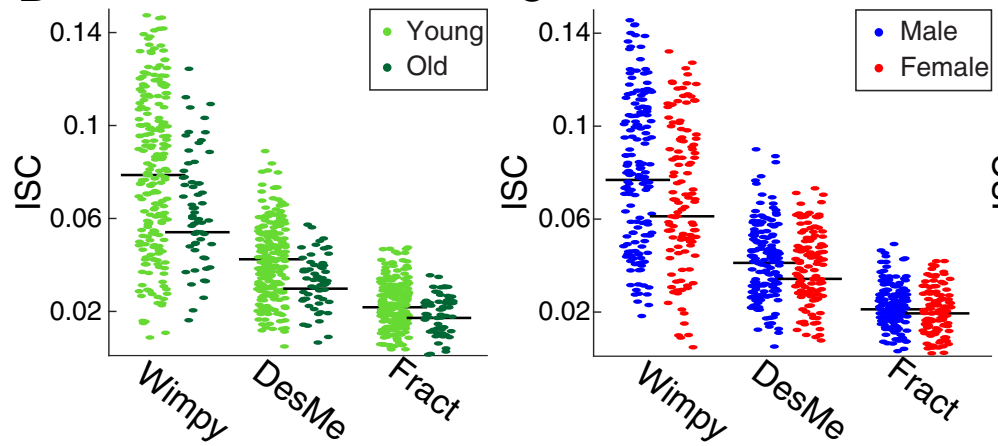

D

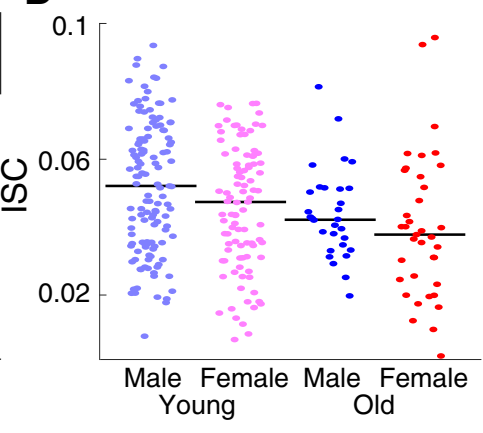

Figure 9. The results from the main study replicated in an independent cohort $(n=303)$. $\boldsymbol{A}$, ISC decreased with age in the replication cohort. ISC was computed for each individual by correlating responses from individual subjects to those from all other subjects (regardless of age and sex) for that stimulus. Correlation values ranged from $r=-0.37$ to $r=-0.44$. Note that for every stimulus, a different number of subjects was available. B, Across all stimuli, ISC was higher for younger subjects (6-14 yr, light green) than it was for older subjects (15-44 yr, dark green) in the replication cohort. For consistency, the split between the ages was consistent between this study and the main study. $\boldsymbol{C}$, Across all stimuli, ISC was higher for males (blue) than females (red) in the replication cohort. For both $\boldsymbol{B}$ and $\boldsymbol{C}$, ISC was computed separately within each age and sex group. Black lines indicate the median. $\boldsymbol{D}$, Sex differences in the young disappeared with age in the replication cohort. Young males were more neurally similar to each other than young females, and this sex difference was absent in the older group. Here, ISC was computed within each sex and age group separately and averaged across all stimuli used in the replication cohort. Black lines indicate the median.

with age. This indicates that patterns of neural activity may potentially increase in their diversity with age as top-down factors relating to the interpretation of naturalistic stimuli develop. Even in the condition where subjects were instructed to maintain a fixed gaze position (Flash), ISC decreased with age. Future studies with fine-grained eye-tracking during EEG could more definitively answer this question.

The observed ISC magnitude changes with age and sex may also be partially dependent on evoked response magnitudes, which typically decrease with age. Although the amplitudes of auditory ERPs and their components decline with age (Goodin et al., 1978; Tomé et al., 2015), other components increase with age (Dinteren et al., 2014) or remain stable across development (Kujawa et al., 2013). Although correlation, as measured by ISC, is in principle insensitive to magnitude, it is possible that weaker stimulus-evoked responses in adults may be overpowered by non-stimulus-related neural activity (i.e., "noise"; Hämmerer et al., 2013). In this case, a smaller fraction of the signal would correlate across adults in comparison with children. To control for the effect of age, the magnitude of SSVEPs was regressed from the ISC. The result indicates that SSVEP amplitude cannot explain the age effect, but it may explain the sex effect, indicating that males have stronger evoked responses than females (Figs. $3 B$ and $6 B)$. However, it is worth noting that ISC and SSVEPs measure very different facets of neural activity. SSVEPs, extracted from early visual processing areas in V1, likely represent low-level visual processes. ISC, on the other hand, may be driven by higher-level cortical areas, since the spatial distributions of the two dominant components (Fig. 7) do not resemble low-level sensory evoked responses. Parallel work indicates that the first component (C1), which captures the majority of the correlated activity, is a supramodal component that is driven by both auditory and visual stimuli (Cohen and Parra, 2016).

It is also possible that ISC decreases with age because adults process the world with more diverse brain activity. In this view, adults have more highly variable stimulusevoked responses, and their neural activity is therefore less similar across subjects. In this case, it would be likely that the dimensionality of neural responses, a measure of their complexity, increases with age (Anokhin et al., 2000; Mcintosh et al., 2008; Vakorin et al., 2013). There was no clear trend indicative of a difference in the dimensionality between the young and old group. However, it does appear that females have more diverse responses than males, a 
result that deserves further exploration and could possibly underlie the reduction in ISC in this group.

The present results appear to be consistent with Campbell et al. (2015), who, using fMRI, also found a decrease of ISC with age. However, whereas we study an age range dominated by development and corresponding improvements in cognitive performance (6-44 and 5-23 yr in each cohort), Campbell et al. (2015) examined a range (18-88 years) that exhibited a deterioration in fluid intelligence and reaction time. These measures correlated with a decrease in ISC. Whereas Cantlon and Li (2013) studied a cohort that was more comparable to ours in (4-25 yr), they found that ISC of fMRI was generally higher among adults (above age 18) than it was in children (below age 11). In total, it appears that ISC as assessed by fMRI increases with development and declines in older age, which potentially opposes our result with EEG. These differences may be due to important methodological discrepancies between these studies and ours. To more definitively establish the effect of age on ISC, more work should be done using both $\mathrm{FMRI}$ and EEG.

The idea that maturity is marked by variability is not new (Campbell et al., 2015). It aligns with theories from neural systems modeling and human studies (Mcintosh et al., 2008; Vakorin et al., 2011). In these models, moderate amounts of noise or variability facilitate efficient responses in complex environments. Increased variability may be the reason for reduced evoked-response magnitudes, since ERPs are obtained by averaging across many events that are inherently sensitive to signal noise. It is therefore possible that the increased variability of evoked responses across trials with age results in reduced ERP magnitudes.

In the age range examined, neural development is a dynamic process. At the macro level, longitudinal structural neuroimaging shows that cortical thinning occurs from childhood through early adulthood, progressing in a caudal to rostral pattern (Gogtay et al., 2004; Giedd et al., 2015). At the micro level, synaptic pruning and myelination, particularly in the frontal lobe, are ongoing during this period (Rakic et al., 1994; Huttenlocher and Dabholkar, 1997; Cox et al., 2016). From a functional perspective, studies of functional connectivity and task-based fMRI suggest that functional maturation tends to follow a "diffuse to focal" pattern (Durston et al., 2006; Grill-Spector et al., 2008; Fair et al., 2009; Kelly et al., 2009) and may correspond to the extraordinary advances in behavior during childhood (Xiao et al., 2016). Speculatively, the decreased ISC strength in older ages may reflect greater interindividual variability that results from the interplay of structural and functional "streamlining" of neural architecture with distinct life experiences (e.g. cortical thinning, synaptic pruning, and diffuse-to-focal shifts in functional patterns). However, one limitation of the present study is that it is cross-sectional rather than longitudinal, it is therefore difficult to make developmental claims based on the age-based differences demonstrated here (Kraemer et al., 2000).

The age-related effect may also be echoed by the sex difference in neural variability. Longitudinal studies have demonstrated that females mature before males in a range of anatomic measures (Lenroot et al., 2007; Lim et al., 2015). However, differences in developmental trajectories between males and females may be complicated by the fact that the sexes ultimately differ in their mature neuroanatomy (Marsh et al., 2008). Here, sex-related differences in neural variability were seen only among younger subjects, suggesting that this is a developmentrelated difference. Prepubescent and early teenage years are marked by sex differences in behavioral maturity that may not be present in later years (Mous et al., 2017). The difference in neural variability may also be due to pubertal stage, since it is known that females reach pubertal maturity 2-3 yr before males (Sisk and Foster, 2004). However, physiologic pubertal stage was not measured here, and it is therefore not possible to determine whether the sex differences were related to this factor.

Among the different stimuli used, the clips from conventional cinema (Wimpy and DesMe) evoked a higher level of ISC than the educational videos (Arith, Fract, and StudT). The Hollywood clips were rich with scene cuts and dynamic visual cues and are therefore expected to elicit strong levels of ISC (Poulsen et al., 2017). However, previous research has also shown that engagement with narrative stimuli modulates ISC, and it is therefore likely that these Hollywood clips are more effective at engaging attention and thus elicit stronger ISC (Dmochowski et al., 2014; Ki et al., 2016; Cohen et al., 2017). Although the ISC differences between age and sex may be due to each cohort's average level of attention, no independent measures of engagement or attention were collected. It is therefore not possible to determine whether the present effects are driven by attention or differences in low-level stimulus features. Most of the videos were aimed at younger audiences (i.e., Despicable Me, Diary of a Wimpy Kid), and older subjects may have therefore been less interested in them. However, this was not uniformly the case, for instance, the video about Fractals (Fract) may have been equally interesting to both children and adults, whereas the Flash stimulus may be equally boring for all ages. Thus, these two stimuli provided an important control for attentional effects on the age-related differences in ISC. Future work may benefit from looking at objective measures of engagement (Cohen et al., 2017) in the different cohorts studied here. An understanding of such factors, and their impact on behavior, may be of relevance to models of media-based addiction (e.g., Internet addiction, pornography addiction), as well as commercial neuroscience enterprises. Regardless, it is of note that the age effect seen for the naturalistic videos was echoed in the SSVEP condition. Because this stimulus should be equally (un)engaging for all ages, this favors an interpretation based on neural maturation rather than attention.

Future work should recruit a larger sample of subjects older than $15 \mathrm{yr}$ to determine whether the age-related decline in ISC observed in later teenage years continues in adulthood, or might even reverse in older age (Grady, 2012; Campbell et al., 2015). Future studies with clinical cohorts could explore the potential link between ISC and behavioral markers of neural development. It is possible that neural variability is not only a marker of maturity, but 
also a marker of neuropsychiatric disorders (Dinstein et al., 2015). The methods used here provide a novel way of assessing such markers under complex, naturalistic conditions.

Overall, the current results regarding intersubject correlation in children and adults are interpreted in the context of neural maturation. Although males are delayed in the development of the neural variability that appears to be a mark of maturity, the data presented here indicate that with normal development they are no different from females as adults. Thus, with maturity, neural function becomes more variable.

\section{References}

Alexander LM, et al. (2017) The Healthy Brain Network Biobank: an open resource for transdiagnostic research in pediatric mental health and learning disorders. bioRxiv doi: https://doi.org/10.1101/ 149369.

Anokhin AP, Lutzenberger W, Nikolaev A, Birbaumer N (2000) Complexity of electrocortical dynamics in children: developmental aspects. Dev Psychobiol 36:9-22. Medline

Ben-Yakov A, Honey CJ, Lerner Y, Hasson U (2012) Loss of reliable temporal structure in event-related averaging of naturalistic stimuli. Neuroimage 63:501-506. CrossRef

Benjamini Y, Hochberg Y (1995) Controlling the false discovery rate: a practical and powerful approach to multiple testing. J R Stat Soc Ser B 57:289-300.

Campbell KL, et al. (2015) Idiosyncratic responding during moviewatching predicted by age differences in attentional control. Neurobiol Aging 36:3045-3055. CrossRef Medline

Cantlon JF, Li R (2013) Neural activity during natural viewing of Sesame Street statistically predicts test scores in early childhood. PLoS Biol 11:1001462. CrossRef

Cohen SS, Henin S, Parra LC (2017) Engaging narratives evoke similar neural activity and lead to similar time perception. Sci Rep 7:1-10. CrossRef

Cohen SS, Parra LC (2016) Memorable audiovisual narratives synchronize sensory and supramodal neural responses. eNeuro 020316.2016.

Cox SR, Ritchie SJ, Tucker-Drob EM, Liewald DC, Hagenaars SP, Davies G, Wardlaw JM, Gale CR, Bastin ME, Deary IJ (2016) Age differences in brain white matter microstructure in UK Biobank $(\mathrm{N}=3,513)$. bioRxiv 51771:1-34.

Dinstein I, Heeger DJ, Behrmann M (2015) Neural variability: Friend or foe? Trends Cogn Sci 19:322-328. CrossRef Medline

Dinteren R, Arns M, Jongsma MLA, Kessels RPC (2014) P300 development across the lifespan: a systematic review and metaanalysis. PLoS One 9:e87347.

Dmochowski JP, Bezdek MA, Abelson BP, Johnson JS, Schumacher EH, Parra LC (2014) Audience preferences are predicted by temporal reliability of neural processing. Nat Commun 5:1-9. CrossRef

Dmochowski JP, Sajda P, Dias J, Parra LC (2012) Correlated components of ongoing EEG point to emotionally laden attention-a possible marker of engagement? Front Hum Neurosci 6:1-9. CrossRef

Durston S, Davidson MC, Tottenham N, Galvan A, Spicer J, Fossella JA, Casey BJ (2006) A shift from diffuse to focal cortical activity with development. Dev Sci 9:1-20. CrossRef Medline

Fair DA, Cohen AL, Power JD, Dosenbach NUF, Church JA, Miezin FM, Schlaggar BL, Petersen SE (2009) Functional brain networks develop from a "local to distributed" organization. PLoS Comput Biol 5:14-23. CrossRef

Giedd JN, Blumenthal J, Jeffries NO, Castellanos FX, Liu H, Zijdenbos A, Paus T, Evans AC, Rapoport JL (1999) Brain development during childhood and adolescence: a longitudinal MRI study. Nat Neurosci 2:861-863. CrossRef Medline
Giedd JN, Raznahan A, Alexander-Bloch A, Schmitt E, Gogtay N, Rapoport JL (2015) Child Psychiatry Branch of the National Institute of Mental Health longitudinal structural magnetic resonance imaging study of human brain development. Neuropsychopharmacology 40:43-49. CrossRef

Gogtay N, Giedd JN, Lusk L, Hayashi KM, Greenstein D, Vaituzis a. C, Nugent TF, Herman DH, Clasen LS, Toga AW, Rapoport JL, Thompson PM (2004) Dynamic mapping of human cortical development during childhood through early adulthood. Proc Natl Acad Sci USA 101:8174-8179. CrossRef

Goodin DS, Squires KC, Henderson BH, Starr A (1978) Age-related variations in evoked potentials to auditory stimuli in normal human subjects. Electroencephalogr Clin Neurophysiol 44:447-458. Medline

Grady C (2012) The cognitive neuroscience of ageing. Nat Rev Neurosci 13:491-505. CrossRef

Grill-Spector K, Golarai G, Gabrieli J (2008) Developmental neuroimaging of the human ventral visual cortex. Trends Cogn Sci 12:152162. CrossRef Medline

Hämmerer D, Li SC, Völkle M, Müller V, Lindenberger U (2013) A lifespan comparison of the reliability, test-retest stability, and signal-to-noise ratio of event-related potentials assessed during performance monitoring. Psychophysiology 50:111-123. CrossRef Medline

Hasson U, Malach R, Heeger DJ (2009) Reliability of cortical activity during natural stimulation. Trends Cogn Sci 14:40-48.

Hasson U, Nir Y, Levy I, Fuhrmann G, Malach R (2004) Intersubject synchronization of cortical activity during natural vision. Science 303:1634-1640. CrossRef Medline

Haufe S, DeGuzman P, Henin S, Arcaro M, Honey CJ, Hasson U, Parra LC (2017) Elucidating relations between fMRI, ECoG and EEG through a common natural stimulus. bioRxiv 207456. Available at: https://doi.org/10.1101/207456.

Hutchison RM, Morton JB (2015) Tracking the brain's functional coupling dynamics over development. 35:6849-6859. CrossRef Medline

Huttenlocher PR, Dabholkar AS (1997) Regional differences in synaptogenesis in human cerebral cortex. J Comp Neur 387:167-178. CrossRef

Kauppi J-P, Jaaskelainen IP, Sams M, Tohka J (2010) Inter-subject correlation of brain hemodynamic responses during watching a movie: localization in space and frequency. Front Neuroinform 4:1-10.

Kelly AMC, Di Martino A, Uddin LQ, Shehzad Z, Gee DG, Reiss PT, Margulies DS, Castellanos FX, Milham MP (2009) Development of anterior cingulate functional connectivity from late childhood to early adulthood. Cereb Cortex 19:640-657. CrossRef

Ki J, Kelly S, Parra LC (2016) Attention strongly modulates reliability of neural responses to naturalistic narrative stimuli. J Neurosci 36:3092-3101.

Kirkorian HL, Anderson DR, Keen R (2012) Age differences in online processing of video: an eye movement study. Child Dev 83:497507. CrossRef Medline

Kraemer HC, Yesavage JA, Taylor JL, Kupfer D (2000) How can we learn about developmental processes from cross-sectional studies, or can we? Am J Psychiatry 157:163-171. CrossRef

Kujawa A, Klein DN, Proudfit GH (2013) Two-year stability of the late positive potential across middle childhood and adolescence. Bio Psychol 94:290-296. CrossRef

Lahnakoski JM, Jääskeläinen IP, Sams M, Nummenmaa L (2017) Neural mechanisms for integrating consecutive and interleaved natural events. Hum Brain Mapping 38:3360-3376.

Langer N, Ho EJ, Alexander LM, Xu HY, Jozanovic RK, Henin S, Petroni A, Cohen S, Marcelle ET, Parra LC, Milham MP, Kelly SP (2017) A resource for assessing information processing in the developing brain using EEG and eye tracking. Sci Data 4:170040 CrossRef

Lenroot RK, Giedd JN (2006) Brain development in children and adolescents: insights from anatomical magnetic resonance imaging. Neurosci Biobehav Rev 30:718-729. CrossRef Medline

Lenroot RK, Gogtay N, Greenstein DK, Wells EM, Wallace GL, Clasen LS, Blumenthal JD, Lerch J, Alex P, Evans AC, Thompson PM, 
Giedd JN (2007) Sexual dimorphism of brain developmental trajectories during childhood and adolescence. Neuroimage 36:10651073. CrossRef

Lim S, Han CE, Uhlhaas PJ, Kaiser M (2015) Preferential detachment during human brain development: Age- and sex-specific structural connectivity in diffusion tensor imaging (DTI) data. Cereb Cortex 25:1477-1489. CrossRef Medline

Lin Z, Chen M, Ma Y (2013) The augmented Lagrange multiplier method for exact recovery of corrupted low-rank matrices. arXiv Available at: http://arxiv.org/abs/1009.5055\%0Ahttp CrossRef.

Marsh R, Gerber AJ, Peterson BS (2008) Neuroimaging studies of normal brain development and their relevance for understanding childhood neuropsychiatric disorders. J Am Acad Child Adolesc Psychiatry 47:1233-1251. CrossRef Medline

Mcintosh AR, Kovacevic N, Itier RJ (2008) Increased brain signal variability accompanies lower behavioral variability in development. PLoS Comput Biol 4:e1000106. CrossRef

Mous SE, et al. (2017) The association of gender, age, and intelligence with neuropsychological functioning in young typically developing children: The Generation R study. Appl Neuropsychol Child 6:22-40. CrossRef

Papenberg G, Hämmerer D, Müller V, Lindenberger U, Li SC (2013) Lower theta inter-trial phase coherence during performance monitoring is related to higher reaction time variability: a lifespan study. Neuroimage 83:912-920. CrossRef

Parra LC, Spence CD, Gerson AD, Sajda P (2005) Recipes for the linear analysis of EEG. Neuroimage 28:326-341. CrossRef Medline
Poulsen AT, Kamronn S, Dmochowski J, Parra LC, Hansen LK (2017) Measuring engagement in a classroom: synchronised neural recordings during a video presentation. Sci Rep 7:1-9. CrossRef

Rakic P, Bourgeois JP, Goldman-Rakic PS (1994) Synaptic development of the cerebral cortex: implications for learning, memory, and mental illness. Prog Brain Res 102:227-243. CrossRef Medline

Sisk CL, Foster DL (2004) The neural basis of puberty and adolescence. Nat Neurosci 7:1040-1047. CrossRef Medline

Tomé D, Barbosa F, Nowak K, Marques-Teixeira J (2015) The development of the $\mathrm{N} 1$ and $\mathrm{N} 2$ components in auditory oddball paradigms: a systematic review with narrative analysis and suggested normative values. J Neural Transm 122:375-391. CrossRef Medline

Vakorin VA, Lippe S, Mclntosh AR (2011) Variability of brain signals processed locally transforms into higher connectivity with brain development. J Neurosci 31:6405-6413. CrossRef

Vakorin VA, McIntosh AR, Mišić B, Krakovska O, Poulsen C, Martinu K, Paus T (2013) Exploring age-related changes in dynamical non-stationarity in electroencephalographic signals during early adolescence. PLoS One 8:e57217. CrossRef

Vanegas MI, Blangero A, Kelly SP (2015) Electrophysiological indices of surround suppression in humans. J Neurophysiol 113:11001109. CrossRef

Xiao Y, Friederici AD, Margulies DS, Brauer J (2016) Longitudinal changes in resting-state fMRI from age 5 to age 6 years covary with language development. Neuroimage 128:116-124. CrossRef 